04.1

\title{
Пылевая плазма в сильно неоднородном магнитном поле
}

\author{
() В.Ю. Карасев, Е.С. Дзлиева, С.И. Павлов, Л.А. Новиков, И.Ч. Машек \\ Санкт-Петербургский государственный университет, Санкт-Петербург, Россия \\ E-mail: plasmadust@yandex.ru, v.karasev@spbu.ru
}

Поступило в Редакцию 20 января 2020 г.

В окончательной редакции 20 января 2020г.

Принято к публикации 23 января 2020г.

\begin{abstract}
Впервые представлены исследования пылевой плазмы, сформированной в тлеющем разряде, в области сильно неоднородного магнитного поля. Неоднородность магнитного поля оценена величиной $2 \cdot 10^{3} \mathrm{G} / \mathrm{cm}$. Обнаружена устойчивая пылевая ловушка. Определены геометрические характеристики плазменно-пылевой структуры, измерена угловая скорость вращения в нескольких сечениях, перпендикулярных оси разрядной трубки. Предложен механизм вращения плазменно-пылевой структуры, выполнены количественные оценки, подтвердившие экспериментальные данные.
\end{abstract}

Ключевые слова: пылевая плазма, неоднородное магнитное поле, тлеющий разряд, пылевая ловушка.

DOI: 10.21883/PJTF.2020.08.49302.18208

Пылевая плазма - низкотемпературная плазма с внедренными в нее макрочастицами микронного и наноразмерного диапазонов, аккумулирующими на поверхности значительный заряд, - обладает рядом уникальных свойств и относится к самостоятельному разделу физики плазмы [1-3]. Первоначально она была обнаружена в технологических камерах, и сегодня существует множество ее технических приложений в плазменных процесcax: травлении, синтезе материалов, в энергетических установках [4,5]. В этом контексте особый интерес имеет исследование объемных плазменно-пылевых образований, локализованных вблизи стенок плазменных установок, при наличии сильного магнитного поля. В работах [6-8] описаны исследования, направленные на изучение особенностей динамики и управления пылевыми структурами. Среди экспериментальных исследований пылевой плазмы в сильном магнитном поле (см., например, [9-11]) только два были проведены с объемными пылевыми структурами [12,13]. Но все исследования были выполнены в однородном магнитном поле. В настоящей работе впервые изучается пылевая плазма в форме объемной пылевой структуры, созданной в резко неоднородном сильном магнитном поле с градиентом порядка $2 \cdot 10^{3} \mathrm{G} / \mathrm{cm}$.

При создании пылевой плазмы в стратах в тлеющем разряде в магнитном поле $[12,13]$ технически сложным моментом является сохранение стабильной пылевой ловушки и удержание стоячей страты в области однородного поля. При увеличении магнитного поля страта существенно вытягивается и смещается в сторону анода. При сканировании пылевой структуры вдоль оси разрядной трубки было обнаружено, что вне соленоида вблизи его торца с анодной части в сильном магнитном поле образуется стоячая страта. В ней формируется объемная пылевая структура, приходящая в достаточно быстрое вращение со скоростью от 5 до $10 \mathrm{rad} / \mathrm{s}$ в зависимости от сорта газа, его давления, радиуса трубки и тока. Угловая скорость направлена противоположно вектору магнитной индукции. Обнаруженная пылевая
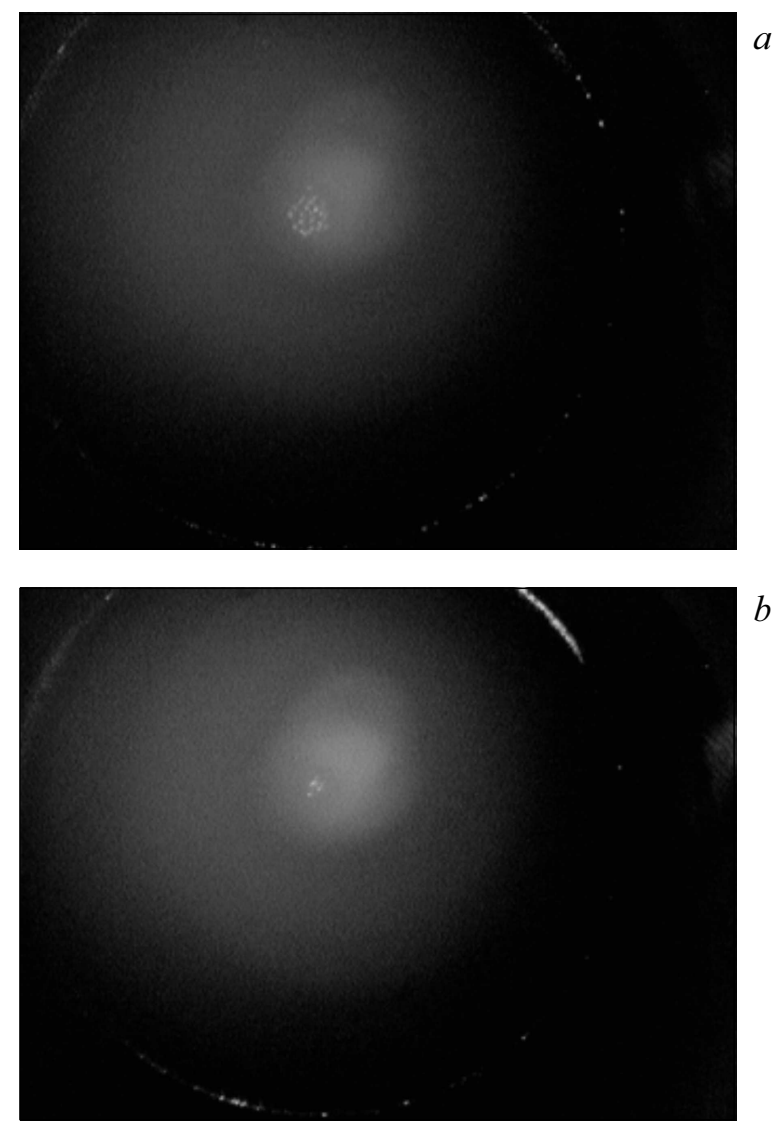

Рис. 1. Изображения горизонтальных сечений плазменнопылевой структуры, имеющих координаты 134 (a) и $110 \mathrm{~mm}$ $(b)$. Вертикальная координата отсчитывается от центра соленоида, создающего магнитное поле. Условия: неон при давлении 0.63 Torr, разрядный ток $1.5 \mathrm{~mA}$, магнитное поле $7500 \mathrm{G}$. Диаметр разрядной трубки (виден как лазерный спекл на фотографии) равен $21 \mathrm{~mm}$. Полидисперсные частицы кварца. 
ловушка представляет собой существенно искаженную страту. Она находится в области сильно спадающего магнитного поля (на торце соленоида от 1000 до $50 \mathrm{G}$ ). Описанная картина формирования и вращения остается стабильной до максимального увеличения магнитного поля в сверхпроводящем соленоиде до $2 \cdot 10^{4} \mathrm{G}$.

На экспериментальной установке, описанной в $[8,12,13]$, были получены следующие результаты. При магнитном поле вблизи торца соленоида до $1000 \mathrm{G}$ формируется плазменно-пылевая ловушка, имеющая коническую форму. На рис. 1 показаны два изображения горизонтальных сечений пылевой структуры, разнесенных на $\sim 25 \mathrm{~mm}$. Сканирование горизонтальных сечений пылевой структуры было проведено в следующих условиях: газ - неон, давление $p=0.63$ Torr, ток разряда $1.5 \mathrm{~mA}$, радиус трубки $10 \mathrm{~mm}$, магнитное поле в центре соленоида 7500 G. По видеозаписям определялась угловая скорость вращения в зависимости от вертикальной координаты (рис. 2). Пылевая ловушка располагалась в резко изменяющемся магнитном поле, пример продольного градиента магнитного поля показан на рис. 3, a. При этом установлено, что в центральной части сечения, где располагается разрядная трубка, радиальный градиент продольного магнитного поля очень мал (рис. 3). Дополнительные измерения радиальной составляющей магнитного поля показали, что она более чем на порядок меньше продольной составляющей поля в области нахождения пылевой структуры. Установившееся значение скорости вращения, например $5 \mathrm{rad} / \mathrm{s}$ (по абсолютной величине) в экстремуме на рис. 2, не изменялось при увеличении магнитного поля в центре соленоида до $2 \cdot 10^{4} \mathrm{G}$ (соответственно на торце соленоида до $2000 \mathrm{G}$ ).

Анализируя возможные причины вращения пылевой плазмы, мы заключили, что наблюдаемое вращение

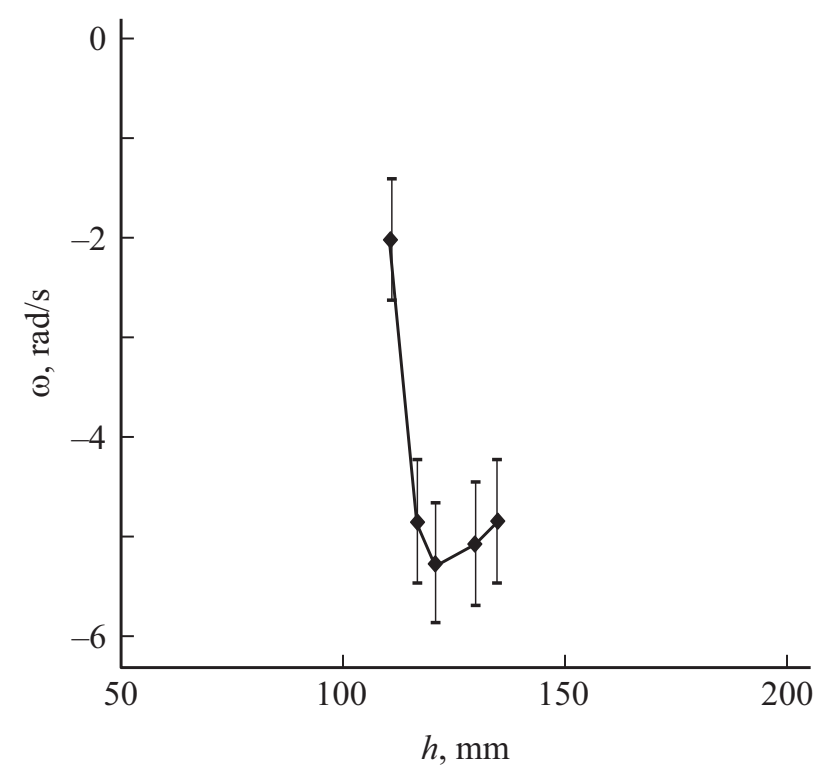

Рис. 2. Зависимость угловой скорости вращения горизонтального сечения плазменно-пылевой структуры от вертикальной координаты. Условия те же, что для рис. 1.
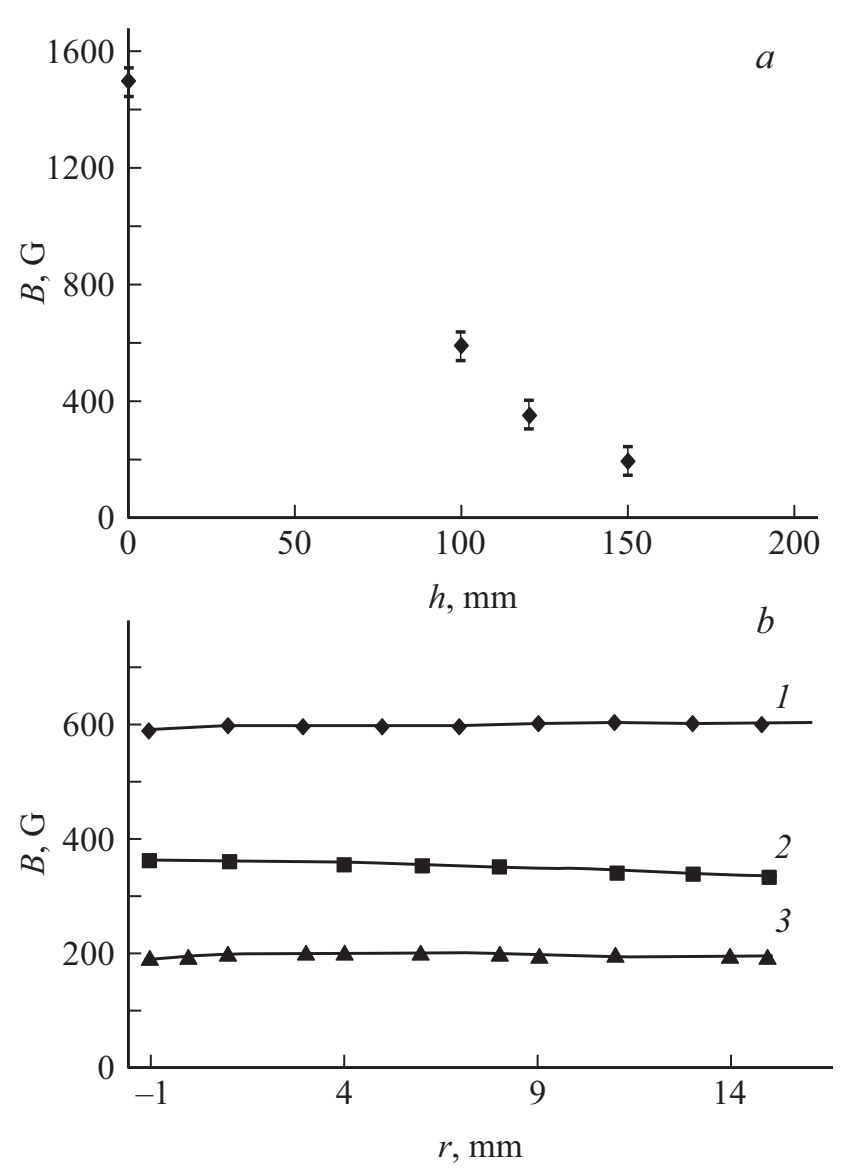

Рис. 3. $a-$ измеренные значения вертикальной составляющей магнитного поля на оси разрядной трубки в области левитации структуры (соответствует рис. 2). Верхний торец соленоида располагается на высоте $h=100 \mathrm{~mm}$. $b$ - измеренные значения вертикальной составляющей магнитного поля в зависимости от радиальной координаты на высоте $h=100(1)$, 120 (2) и $150 \mathrm{~mm} \mathrm{(3).}$

вызвано силой Ампера при действии продольной составляющей магнитного поля на появившуюся радиальную компоненту тока вблизи торца соленоида. Выражение для угловой скорости вращения можно получить, приравнивая момент силы Ампера $M_{B}$ к моменту силы вязкого трения газа разряда о стенку разрядной трубки. Величина $M_{B}$ определяется выражением

$$
M_{B}=\left(\mathbf{j}_{r} \times \mathbf{B}\right) S \Delta r r_{a v},
$$

где $\mathbf{j}_{r}$ - радиальная составляющая вектора объемной плотности тока, $S$ - площадь цилиндрической поверхности радиуса $r_{a v}=\left(r_{1}+r_{2}\right) / 2, \Delta r=r_{2}-r_{1}-$ изменение радиуса поперечного сечения разряда.

Величина $M_{\eta}$ определяется выражением

$$
M_{\eta}=2 \pi \eta \omega r_{a v} R_{0} h
$$

где $R_{0}$ - радиус трубки, $\omega-$ угловая скорость вращения газа, $\eta-$ вязкость газа. 
Скорость вращения газа (и сечения пылевой структуры) дается выражением

$$
\omega_{\eta}=\frac{j_{r} B r_{a v} \Delta r}{\eta R_{0}}
$$

Численная оценка для среднего по высоте сечения дает от 2.5 до $10 \mathrm{rad} / \mathrm{s}$ (по абсолютной величине) в зависимости от предположения о радиальной составляющей тока.

Также можно пояснить наличие экстремума на зависимости угловой скорости от высоты структуры (рис. 2). Ниже $100 \mathrm{~mm}$ (канал тока сжат) и выше примерно $130 \mathrm{~mm}$ (канал тока расширен) радиальной составляющей тока нет. Между этими точками и работает механизм раскручивания газа, приводящий среднее сечение структуры $125 \mathrm{~mm}$ в наиболее быстрое вращение.

Таким образом, в работе исследовалась пылевая плазма в тлеющем разряде в сильно неоднородном магнитном поле. Обнаружена устойчивая пылевая ловушка. Проведены количественные измерения скорости вращения, геометрии пылевой структуры и неоднородности магнитного поля. Предложен механизм вращения этой пылевой структуры, выполнены количественные оценки, подтвердившие экспериментальные данные.

\section{Благодарности}

Часть расходных материалов и оборудования были получены по программе модернизации материальной и технической базы научных исследований Санкт-Петербургского государственного университета (СПбГУ) „Полимерные частицы в пылевой плазме“.

\section{Финансирование работы}

Работа выполнена при поддержке Российского научного фонда (грант № 18-72-10019).

\section{Конфликт интересов}

Авторы заявляют, что у них нет конфликта интересов.

\section{Список литературы}

[1] Complex and dusty plasmas: from laboratory to space / Eds V.E. Fortov, G.E. Morfill. N.Y.: Taylor \& Francis Group, 2010. $440 \mathrm{p}$.

[2] Tsytovich V.N., Morfill G.E., Vladimirov S.V., Thomas H.M. Elementary physics of complex plasmas. N.Y.: Springer, 2008. $370 \mathrm{p}$.

[3] Introduction to complex plasma / Eds M. Bonitz, N. Horing, P. Ludwig. Berlin-Heidelberg: Springer-Verlag, 2010. 443 p.

[4] Vladimirov S.V., Ostrikov K., Samarian A.A. Physics and applications of complex plasmas. London: Imperial College Press, 2005. $439 \mathrm{p}$.

[5] Цытович В.Н., Морфилл Г.Е., Томас Х. // Физика плазмы. 2004. T. 30. № 10. C. 877-929.
[6] Паль А.Ф., Рябинкин А.Н., Серов А.О., Филиппов А.В. // Письма в ЖТФ. 2014. Т. 40. В. 24. С. 112-119.

[7] Недоспасов А.В., Ненова Н.В. // Письма в ЖТФ. 2011. T. 37. B. 18. C. 26-30.

[8] Дзлиева Е.С., Новиков Л.А., Павлов С.И., Карасев В.Ю. // Письма в ЖТФ. 2018. Т. 44. В. 19. С. 66-71.

[9] Thomas E., Jr., Lynch B., Konopka U., Merlino R.L., Rosenberg M. // Phys. Plasmas. 2015. V. 22. P. 030701 (1-4).

[10] Schwabe M., Konopka U., Bandyopadhyay P., Morfill G.E. // Phys. Rev. Lett. 2011. V. 106. P. 215004.

[11] Melzer A., Kruger H., Schutt S., Mulsow M. // Phys. Plasmas. 2019. V. 26. P. 093702.

[12] Dzlieva E.S., Dyachkov L.G., Novikov L.A., Pavlov S.I., Karasev V.Yu. // Europhys. Lett. 2018. V. 123. P. 15001.

[13] Dzlieva E.S., Dyachkov L.G., Novikov L.A., Pavlov S.I., Karasev V.Yu. // Plasma Sources Sci. Technol. 2019. V. 28. P. 085020. 